\title{
Genovese, E. and Galper, J. (eds): Guide to the Evaluation of Functional Ability: How to Request, Interpret, and Apply Functional Capacity Evaluations
}

\author{
American Medical Association, United States of America, 2009, 530 pp, \$75.00 \\ (softbound)
}

Bruce A. Barron

Published online: 30 December 2010

(C) Springer Science+Business Media, LLC 2010

The use and application of functional capacity evaluations (FCEs) have become relatively commonplace in the occupational environment. Although several types of FCEs exist, e.g. pre-offer, post-offer, assessment of injured worker capacity, fitness-for-duty, and return-to-work, the medical reasons for ordering a FCE can be categorized across the following three domains: injury prevention, occupational injury or illness management, and chronic injury or illness management. During the past two decades, a number of FCE protocols have been developed and used by specialists who perform such tests. This has created substantial variability in terms of test safety, utility, practicality, reliability, and validity. Given these limitations and a general lack of standardization in functional ability testing, publication of the Guide to the Evaluation of Functional Ability: How to Request, Interpret, and Apply Functional Capacity Evaluations (Guide) could not have been more timely or more desirable. The stated goals of the book are clearly delineated in its title.

The Guide is a well-referenced and evidenced-based review of the existing FCE literature. The contents of the book address the various aspects of FCE testing that healthcare providers and other stakeholders need to understand in order to request, critically assess, interpret, and apply these specialized tests of functional ability. The first several chapters introduce basic concepts such as general testing principles, reasons to test, and types of tests. Subsequent chapters expound upon basic concepts and include more detailed discussions that encompass a variety of topics, e.g. dynamic material handling, upper extremity and trunk testing, static testing, evaluation of aerobic capacity, and others. The chapters that discussed evaluee effort, chronic pain, clinician and insurer application of FCE results, and legal issues were particularly compelling due to the multitude of controversies that engulf these aspects of functional capacity testing. The authors clearly delineated the pitfalls of functional ability testing and in particular, the limited predictive power of FCEs in the real world. The one major criticism of the book was the tendency for authors to define the terms reliability and validity in their respective chapters. This practice led to superfluous redundancy. Future editions of the book should consider defining these terms at the outset rather than repeating the definitions in applicable chapters.

The Guide is a comprehensive and evidence-based stateof-the-art book that will assist practitioners in knowing when to order FCEs and how to critically assess, interpret, and apply the outcomes of FCEs in a variety of situations. Although functional capacity tests are scientifically-driven, the Guide clarifies their clinical, analytical, and legal limitations. This book would certainly be a useful resource to a variety of stakeholders including but not limited to occupational health professionals, physical and occupational therapists, human resource professionals, claims examiners, disability managers, lawyers, and others involved in the hiring process or the management of ill or injured workers.
B. A. Barron ( $\square)$

Division of Occupational and Environmental Medicine,

University of Rochester School of Medicine and Dentistry,

Rochester, NY, USA

e-mail: bruce_barron@urmc.rochester.edu 